MATHEMATICAL ASSOCIATION

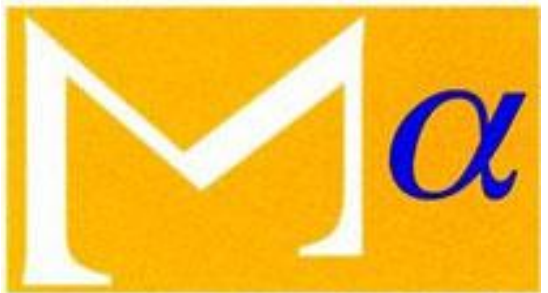

supporting mathematics in education

180. Note on Simson's Line

Author(s): E. P. Rouse

Source: The Mathematical Gazette, Vol. 3, No. 54 (Dec., 1905), pp. 261-262

Published by: Mathematical Association

Stable URL: http://www.jstor.org/stable/3603878

Accessed: 16-01-2016 16:04 UTC

Your use of the JSTOR archive indicates your acceptance of the Terms \& Conditions of Use, available at http://www.jstor.org/page/ info/about/policies/terms.jsp

JSTOR is a not-for-profit service that helps scholars, researchers, and students discover, use, and build upon a wide range of content in a trusted digital archive. We use information technology and tools to increase productivity and facilitate new forms of scholarship. For more information about JSTOR, please contact support@jstor.org. 
180. [K. 2. a.] Note on Simson's Line.

$A, B, C, D$ are points on a circle, centre $O$, radius $R ; K, k$ orthocentres of the triangles $A B C, D B C$.

Then $A K, D k$ are parallel and each equal to twice the perpendicular from $O$ on $B C$, hence $A K k D$ is a parallelogram, and its diagonals bisect each other in $G$.

The rectangular hyperbola through $A, B, C, D$ must also pass through $K$ and $k$; so that $G$ is its centre, and the nine-point circles of the triangles $A B C, D B C, A B D, A C D$ intersect in $G$.

If $L M N, l m n$ be the Simson lines of $D$ and $A$ with respect to the triangles $A B C, D B C$ respectively, we may assume as known that $L M N$ bisects $D K$, i.e. it passes through $G$.

Similarly the pedal lines of $A, B, C$ with respect to the triangles $D B C$, $A C D, A B D$ also pass through $G$.

Let $B A, C D$ meet in $E$; and $B C, A D$ meet in $F$.

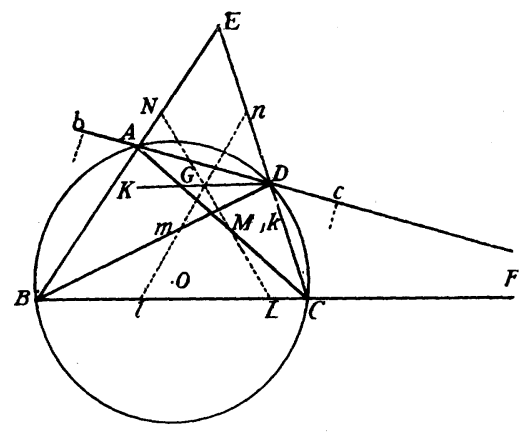

Now $D N A=A n D=90^{\circ}, \therefore A N n D$ is cyclic, and $N n, A D$ are antiparallel with respect to $E A B, E D C$. Hence $N n$ is parallel to $B C$, and in the same way $M m$ is parallel to $B C$.

The perpendicular from $G$ on $B C$ bisects $L l ; \therefore G L=G l, G M=G m$, and $G N=G n$.

Thus the four Simson lines not only co-intersect in $G$, but are all of the same length, and have their segments measured from $G$ to the sides of the respective triangles all equal to one another.

Let $B b, C c$ be perpendiculars to $A D$; then

$$
G b=G c=G l=G L .
$$

Thus $G$ is the circum-centre of the triangle $l b c$, which is similar to $A B C$ (for $\angle l b c=\angle l B A$ and $\angle l c b=\angle B C A$ ).

Hence $G$ is the centroid of masses $\sin 2 A, \sin 2 B, \sin 2 C$ at $l, b, c$ respectively.

But

$$
\angle A b m=\angle A B D=\angle A C D=\angle A c n \text {. }
$$

$\therefore b m$ is parallel to $c n$.

Thus $G$ is also the centroid of masses $\sin 2 A, \sin 2 B, \sin 2 C$ at $l, m, n$ respectively, or (as the respective distances from $G$ are equal) of those masses at $L, M, N$. Similar results hold for the other similar lines.

Lastly, let us take the proposition for which Mr. R. F. Davis (Math. Gazette, No. 48, p. 116) desires a geometrical meaning.

Suppose $A, B, C$ fixed and $D$ variable. 


$$
\begin{aligned}
\Sigma O L^{2} \sin 2 A & =O G^{2} \sum \sin 2 A+\Sigma G L^{2} \sin 2 A \\
& =O G^{2} \sum \sin 2 A+\Sigma G l^{2} \sin 2 A \\
& =\Sigma O l^{2} \sin 2 A \\
& =R^{2} \Sigma \sin 2 A-B l . l C \sin 2 A-B m . m D \sin 2 B \\
& \quad+C n . n D \sin 2 C .
\end{aligned}
$$

But $B l . l C \sin 2 A=2 A B \cdot \cos B \cdot A C \cdot \cos C \sin A \cos A=8 k^{2} \cdot I l \sin A \cdot \Pi \cos A$,

$B m . m D \sin 2 B=2 A B \cos A B D \cdot A D \cos A D B \cdot \sin B \cos B$

$$
\begin{aligned}
& =2 A C \cdot \cos A C D \cdot A D \cdot \cos A D n \cdot \sin C \cdot \cos C \\
& =C n \cdot n D \sin 2 C \cdot
\end{aligned}
$$

Thus

$$
\sum O L^{2} \sin 2 A=4 R^{2} \Pi \sin A(1-2 \Pi \cos A) .
$$

E. P. Rouse.

181. [L'. 2. b.] A solution, not by elliptic functions, is wanted of the following: Given 5 lines $a b c d e$ in a plane, it is known that the pairs of points

$$
a b, c e ; b c, d a ; c d, e b ; d e, a c ; e a, b d
$$

are in a collineation. Prove that the fixed triangle of this collineation is self-polar as to both the conic on $a b, b c, c d$, de, ea and the conic on $a c, c e, e b, b d, d a$.

J. Morley.

\section{2. [K.] Proofs of Euler's Theorem, etc.}

Lemma :-The resultant of successive reflexions in two planes intersecting at an angle $\alpha$ and in a line $l$ is a rotation through $2 \alpha$ about $l$.

To find the resultant of a rotation through $\alpha$ about an axis $O A$ followed by a rotation through $\beta$ about an axis $O B$.

Take $O C$ such that the angle between the planes $O A B$ and $O A C=\frac{1}{2} \alpha$ and the angle between $O B C, O B A=\frac{1}{2} \beta$. Then the required resultant $\equiv$ the resultant of successive reflexions in $O A C, O A B, O B A, O B C \equiv$ the resultant of successive reflexions in $O A C, O B C \equiv$ a rotation about $O C$ through twice the angle between $O C B, O C A$.

To reduce any displacement of a rigid body to its simplest form.

(1) Let $A, B, \ldots$ be the initial and $\alpha, \beta, \ldots$ the final positions of a set of parallel plaues. Then it is readily shown that $A$ and $\alpha, B$ and $\beta, \ldots$ meet on a fixed plane $p$. Let $A^{\prime}, B^{\prime}, \ldots$ and $\alpha^{\prime}, \beta^{\prime}, \ldots$ be the initial and final positions of a second set of parallel planes, and let $p^{\prime}$ be the locus of the intersections of $A^{\prime}$ and $\alpha^{\prime}, B^{\prime}$ and $\beta^{\prime}, \ldots$ Let $S$ be any point on the intersection $l$ of $p$ and $p^{\prime}$ and let $M, \mu, M^{\prime}, \mu^{\prime}$ be the planes through $S$ parallel to $A, \alpha$, and $A^{\prime}, \alpha^{\prime}$. Lei $l$ and $S$ be moved by the displacencent to $\lambda$ and $\sigma$. Since $S$ lies on $M$ and $M^{\prime}, \sigma$ lies on $\mu$ and $\mu^{\prime}$. Hence every point of $l$ is moved parallel to the intersection of $\alpha$ and $\alpha^{\prime}$, and therefore $l$ and $S$ are moved to $\lambda$ and $\sigma$ either (i) by a translation, or (ii) by a rotation through some point $O$ on $l$. In either case the displacement of the body is completed by a rotation about $\lambda$. Hence the total displacement is equivalent (i) to a translation followed by a rotation, i.e. in general to a screw ; (ii) to two rotations about lines through $O$, i.e. to a rotation.

(2) Let two points of the body whose final positions are $A^{\prime}$ and $B^{\prime}$ be brought to $A$ and $B$ by a translation which brings any one point to its final position $O$. Let $\beta$ be the reflexion of $B$ in the plane $p$ bisecting $A A^{\prime}$ at right angles, and let the plane $q$ bisect $B^{\prime} \beta$ at right angles. Then $p$ passes through $O$ since $O A=O A^{\prime}$, and $q$ passes through $O$ and $A^{\prime}$ since $O \beta=O B=O B^{\prime}$ and $A^{\prime} \beta=A B=A^{\prime} B^{\prime}$. Now the points $O, A, B$ are brought to $O, A^{\prime}, B^{\prime}$ by successive reflexions in $p$ and $q$, i.e. by a rotation about the intersection of $p$ and $q$. But three points determine the position of a body, and therefore the displacement is equivalent to a translation followed by a rotation.

These proofs can be modified to suit the case in which the initial and final positions of a figure are enantiomorphous.

HAROLD HILTON. 